\title{
Novos registros de Nitella (Chlorophyta, Characeae) para regiões dos Estados de Mato Grosso e Mato Grosso do Sul, Brasil
}

\author{
Norma Catarina Bueno ${ }^{1,4}$, Thamis Meurer ${ }^{1}$, Stefania Biolo ${ }^{2}$ e Carlos Eduardo de Mattos Bicudo ${ }^{3}$ \\ Recebido: 21.12.2010; aceito: 28.07.2011
}

\begin{abstract}
New records of Nitella (Chlorophyta: Characeae) in Mato Grosso and Mato Grosso do Sul States, Brazil). Charophyceae (Chlorophyta) are a unique class of algae due their vegetative structure and macroscopic gametangia. The

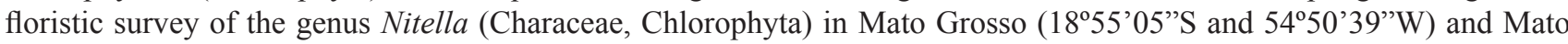
Grosso do Sul States $\left(19^{\circ} 12^{\prime} 03^{\prime \prime}\right.$ S and $\left.57^{\circ} 35^{\prime} 32^{\prime \prime} \mathrm{W}\right)$, was performed by analyzing specimens from SP, HMS and CPAP herbarium specimens, and resulted in the identification, description and illustration of seven species: Nitella acuminata, $N$. axillaris, $N$. flagellifera, $N$. gollmeriana, $N$. intermedia, $N$. mucronata and $N$. tenuissima.
\end{abstract}

Key words: Charophyceae, floristic inventory, macroalgae, taxonomy

RESUMO - (Novos registros de Nitella (Chlorophyta, Characeae) para os Estados de Mato Grosso e Mato Grosso do Sul, Brasil). As Charophyceae (Chlorophyta) representam uma classe única de algas devido à sua estrutura vegetativa e porte macroscópico dos gametângios. O inventário florístico do gênero Nitella (Characeae, Chlorophyta) nos Estados de Mato

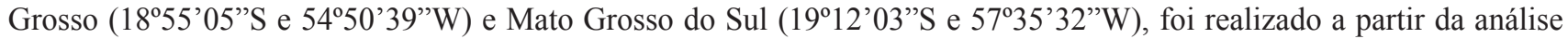
espécimes provenientes dos herbários SP, HMS e CPAP e resultou na identificação, descrição e ilustração de sete espécies: Nitella acuminata, $N$. axillaris, $N$. flagellifera, $N$. gollmeriana, $N$. intermedia, $N$. mucronata e $N$. tenuissima .

Palavras-chave: Charophyceae, inventário florístico, macroalga, taxonomia

\section{Introdução}

As Charophyceae constituem uma classe única de algas graças ao seu porte macroscópico e à grande complexidade morfológica, tanto da organização do talo quanto das estruturas dos gametângios masculino e feminino. O gênero Nitella é representado por macroalgas eretas, sésseis, ecorticadas, eixo principal diferenciados em nós e entrenós, com ramos e râmulos e a presença de gametângios isolados ou em capítulos.

Informações sobre a ocorrência do gênero Nitella nos Estados de Mato Grosso e Mato Grosso do Sul são escassas. Entre os trabalhos estão Braun \& Nordstedt (1882) com o registro de Nitella subglomerata $[=N$. acuminata var. subglomerata (A. Braun) T.F. Allen] para Cáceres e para o rio Paraguai (Mato Grosso) e Bicudo \& Yamaoka (1978) com a citação de Nitella subglomerata para Brasilândia e Cuiabá
(Mato Grosso). Pott et al. (1992) registraram a ocorrência de Nitella cernua A. Braun e N. furcata (Roxburgh ex Bruzelius) C. Agardh em lagoas do Pantanal do Abobral (Mato Grosso do Sul).

Bueno \& Bicudo (1997) identificaram seis espécies, quatro subespécies, três variedades e cinco formas taxonômicas para o Mato Grosso do Sul, sendo elas: Nitella acuminata, $N$. cernua, $N$. furcata subsp. flagellifera, N. furcata subsp. furcata var. sieberi f. microcarpa, N. furcata subsp. furcata var. sieberi f. sieberi, $N$. furcata subsp. mucronata var. mucronata f. mucronata, N. furcata subsp. mucronata var. mucronata f. wrightii, N. gollmeriana, $N$. subglomerata e $N$. translucens subsp. translucens var. axillaris f. axillaris.

O presente trabalho tem como objetivo o levantamento florístico da flora carofítica dos Estados do Mato Grosso e Mato Grosso do Sul - Brasil.

1. Universidade Estadual do Oeste do Paraná, Caixa Postal 711, 85814-110 Cascavel, PR, Brasil

2. Universidade Estadual de Maringá, NUPELIA, Bloco G-90, Av. Colombo 5790, 87020-900 Maringá, PR, Brasil

3. Instituto de Botânica, Núcleo de Pesquisa em Ecologia, Caixa Postal 68041, 04045-972 São Paulo, SP, Brasil

4. Autor para correspondência: norma.bueno@unioeste.br 


\section{Material e métodos}

Foram analisadas 29 amostras provenientes de coleções dos herbários SP (Instituto de Botânica, São Paulo), HMS (Herbário de Mato Grosso do Sul/ Embrapa Gado de Corte, Campo Grande, Mato Grosso do Sul) e CPAP (Centro de Pesquisa Agropecuária do Pantanal/Embrapa Pantanal, Corumbá, Mato Grosso do Sul). Os registros dos herbários continham informações de localização, porém careciam de informações a respeito das características físicas e químicas dos locais de amostragem.

O preparo das lâminas para estudo e identificação taxonômica dos materiais seguiu método descrito em Bicudo (1974) e Bicudo \& Menezes (2006). A classificação seguiu Krause (1997) e as seguintes literaturas foram consultadas para as identificações e discussões dos espécimes: Bicudo (1974, 1977, 1979), Bueno \& Bicudo (1996), Bueno et al. (2009), Groves \& Groves (1911), Moore (1986), Picelli-Vicentim et al. (2004), Proctor et al. (1971), Vieira Junior et al. (2003) e Wood \& Imahori (1964, 1965). Foram adotadas as seguintes abreviaturas: alt. $=$ altura; compr. $=$ comprimento; diâm. $=$ diâmetro; larg. $=$ largura.

\section{Resultados}

Nitella acuminata A. Braun ex Wallman, Försök Syst. Char.: 35. 1853.

Figuras 1 A-G

Plantas monóicas, $20 \mathrm{~cm}$ alt., incrustação calcárea ausente. Caulóide 631,5-989 ㅆm diâm. Entrenós mais longos que o comprimento dos râmulos, 1-4 cm. Râmulos monomórficos, 6-9, 1-furcados, 1-5 cm compr. $\times 206,9-800 \mu \mathrm{m}$ diâm., raios primários 6-9, 300-1632 $\mu \mathrm{m}$ compr. × 122-306 $\mu \mathrm{m}$ diâm., 0,6 vezes o comprimento do râmulo. Dáctilos 2-5, 1-celulados, 1073-4756 $\mu \mathrm{m}$ compr. $\times 110-402 \mu \mathrm{m}$ diâm. Capítulos ausentes. Gametângios conjuntos, sésseis, na base dos dáctilos. Núculas (1-)2 por nó, 283-578 $\mu$ m compr. $\times 168,5-517 \mu$ m diâm., convoluções 9, corônula 26,5-47 $\mu \mathrm{m}$ compr. $\times 44,7-63,4 \mu \mathrm{m}$ diâm., persistente. Oósporos 219-494 $\mu \mathrm{m}$ compr. $\times$ 202-397 $\mu \mathrm{m}$ diâm., estrias 6 , fossa 45-50 $\mu \mathrm{m}$ larg., parede finamente granulada. Glóbulos 167-258 $\mu \mathrm{m}$ diâm., escudos 8, triangulares.

Primeiro registro da espécie para o Estado de Mato Grosso, tendo sido registrada para o Mato Grosso do Sul por Bueno \& Bicudo (1997).
Distribuição no Brasil: Mato Grosso do Sul (Bueno \& Bicudo 1997), Paraná (Thomaz et al. 2003), Rio de Janeiro (Bicudo 1969, Bicudo \& Yamaoka 1978), São Paulo (Picelli-Vicentim 1990, Bicudo 1969, Bicudo \& Yamaoka 1978, Picelli-Vicentim \& Bicudo 1993, Picelli-Vicentim et al. 2004), Rio Grande do Sul (Prado 2003).

Material examinado: BRASIL. Mato Grosso: Brasilândia, 22-IX-1972, D.M. Vital s.n. (SP116358); Cuiabá, 13-I-1893, G.A.N. Malme s.n. (SP114566); 2-IX-1891 S.L.M. Moore s.n. (SP114748); Poconé, 28-II-1996, N.C. Bueno 559 (CPAP10323); 13-V-1995, V.J. Pott 2704 (CPAP13998). Mato Grosso Do Sul: Bela Vista, 19-IV-2005, V.J. Pott et al. 7707 (HMS9250); Corumbá, 6-XII-1894, G.A.N. Malme s.n. (SP114569); 30-IX-1979, C.E.M. Bicudo s.n. (SP155090); Rochedo, 25-I-1979, D.M. Vital (SP152601).

Nitella axillaris A. Braun, Monat. der Deutschen Akad. der Wiss. zu Berlin 1858: 356. 1858.

Figuras $1 \mathrm{H}-\mathrm{M}$

Plantas monóicas, râmulos verticilados dimórficos. Râmulos estéreis bem desenvolvidos, 6-8, 1-2 cm compr., simulando simples, porém 1-furcados. Râmulos férteis formando capítulos, 1-2-furcados, raios primários até 0,5 vezes o comprimento do râmulo, dáctilos 2-4, 2-celulados, 168-358 (-540) $\mu \mathrm{m}$ compr. $\times$ 74-83 $\mu \mathrm{m}$ diâm. Capítulos axilares, 2-3 por verticilo, pedunculados ou não, 1,7-2 mm diâm., envoltos em muco. Núculas 1-3 por nó, 291,5-449 $\mu \mathrm{m}$ compr. $\times$ 291,5-358 $\mu \mathrm{m}$ diâm., convoluções 8-9, corônula 43-45 $\mu \mathrm{m}$ compr. $\times$ 41-55 $\mu \mathrm{m}$ diâm. Glóbulos 108-200 $\mu \mathrm{m}$ diâm., escudos 8 , triangulares.

Nitella axillaris A. Braun foi tratada como uma variedade de Nitella translucens por Wood \& Imahori (1965). Entretanto, os oósporos de N. translucens (John \& Moore 1987) são alongados, ovóides, apresentam ornamentações finamente reticuladas e 5-6 estrias, enquanto os oósporos de $N$. axillaris (Sakayama et al. 2002) são ovóides e exibem ornamentação da parede do oósporo fortemente reticulada e 6-7 estrias. Análises filogenéticas moleculares demonstraram que $N$. axillaris e $N$. translucens são espécies distintas (Sakayama et al. 2004).

O táxon havia sido citado para o Estado do Mato Grosso do Sul como uma variedade de Nitella translucens para as sub-regiões do Pantanal do Abobral, Nabileque e Nhecolândia (Bueno \& 

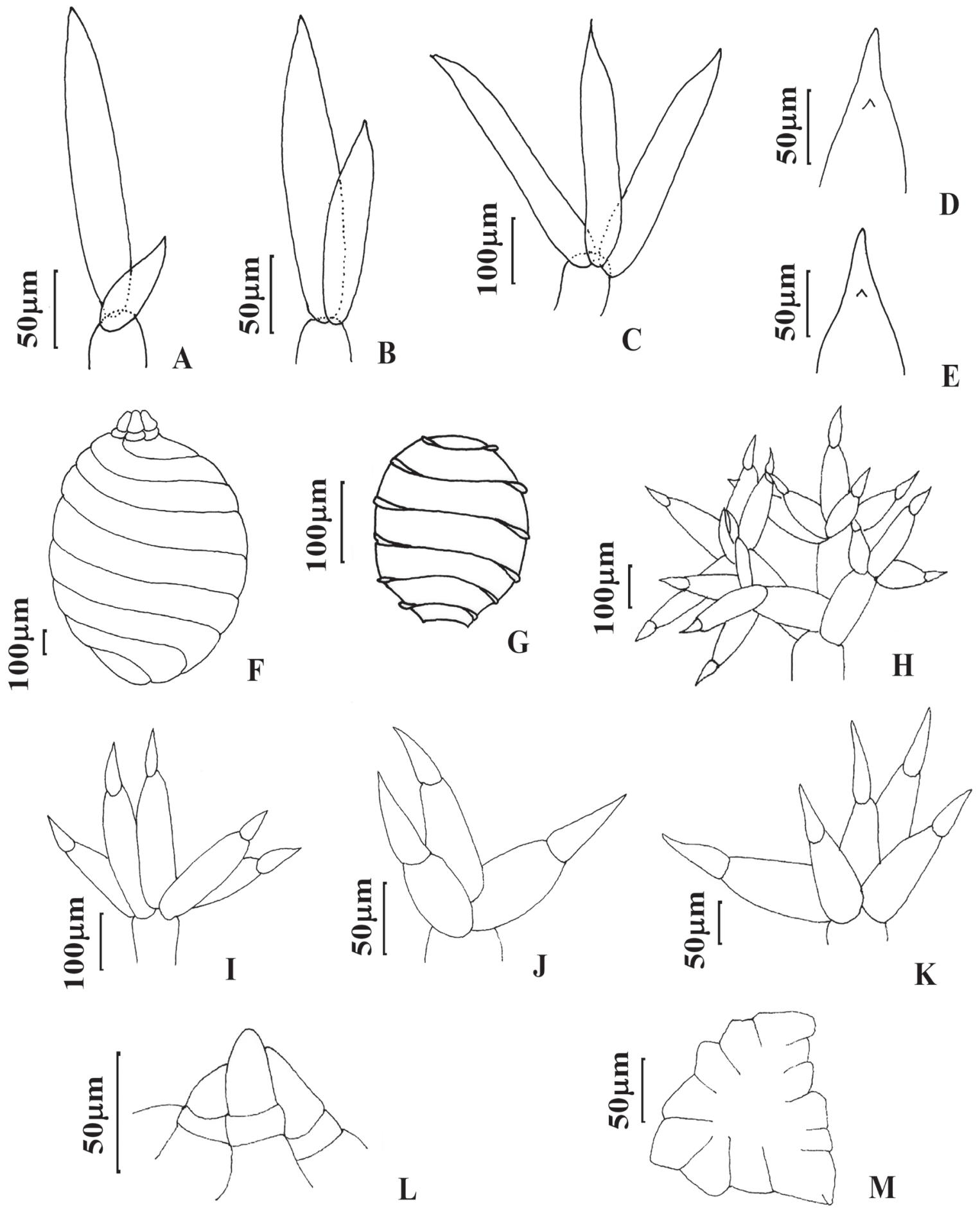

Figura 1. Espécies de Nitella do Mato Grosso e Mato Grosso do Sul, Brasil. A-G. Nitella acuminata. A-C. Dáctilo 1-celulado. D-E. Ápice dos dáctilos. F. Núcula. G. Oósporo. H-M. Nitella axillaris. H. Verticilo estéril. I-K. Dáctilos mucroniformes 2-celulados. L. Corônula. M. Escudo triangular.

Figure 1. Nitella species of Mato Grosso and Mato Grosso do Sul, Brazil. A-G. Nitella acuminata. A-C. One-celled dactyl. D-E. Dactyl apex. F. Oogonia. G. Oospore. H. Nitella axillaris. H-M. Sterile whorl. I-K. Mucroniform, 2-celled dactyls. L. Coronula. M. Triangular scute. 
Bicudo 1997). No presente trabalho o táxon é tratado como espécie, segundo classificação adotada e sua distribuição é ampliada representando portanto novo registro para as regiões de Corumbá e Porto Murtinho, não amostradas no trabalho de Bueno \& Bicudo (1997) realizado no Pantanal (tabela 1). Os exemplares ora analisados apresentaram ligeiras variações métricas no limite inferior do comprimento e diâmetro da núcula $(290 \mu \mathrm{m})$, bem como do diâmetro do glóbulo $(108 \mu \mathrm{m})$. Distribuição no Brasil: [= Nitella translucens (Persoon) C. Agardh emend. R.D. Wood subsp. translucens var. axillaris (A. Braun) R.D. Wood f. axillaris]. Mato Grosso do Sul (Bueno \& Bicudo 1997), Pernambuco (Wood \& Imahori 1965), São Paulo (Picelli-Vicentim 1990, Picelli-Vicentim \& Bicudo 1993, Picelli-Vicentim et al. 2004), Rio Grande do Sul (Prado 2003).

Material examinado: BRASIL. MATo Grosso do SuL: Corumbá, 7-V-1992, N.C. Bueno 319 (CPAP9279); 30-VII-1992, N.C. Bueno 465 (CPAP10229); Porto Murtinho, 26-X-2004, V.J. Pott 7102 (HMS7972).

Nitella flagellifera J. Groves \& G.O. Allen, Journal of Botany, British and Foreing 65: 337. 1927.

Figuras 2 A-I

Plantas monóicas, 10-20 cm alt., incrustação calcárea ausente. Caulóide 324-508 $\mu \mathrm{m}$ diâm. Entrenós 1-2 vezes o comprimento dos râmulos verticilados, 2-3,5 cm compr. Râmulos estéreis 5-7, 2-3-furcados, 1,7-2 cm compr. $\times 200 \mu \mathrm{m}$ diâm., raios primários 5-7, $0,6 \mathrm{~cm}$ compr. $\times 442 \mu \mathrm{m}$ diâm., raios secundários 5-7, 1 central de tamanho variado, substituído ou não por glóbulo, raios terciários $6-7$, raios quaternários 4; dáctilos 4, 2-celulados, 340-5100 $\mu \mathrm{m}$ compr. $\times 52-210,5 \mu \mathrm{m}$ diâm., geralmente alongados, célula subterminal cilíndrica ou quase cilíndrica, célula terminal cônica, ápice agudo. Râmulos férteis 5-7, 2-4-furcados, 7,5-22 mm compr. $\times 110,5 \mu \mathrm{m}$ diâm., dáctilos 2-4, 2-celulados, 189-1182 $\mu \mathrm{m}$ compr. $\times 63-147 \mu$ m diâm., geralmente alongados, ápice agudo. Capítulos ausentes. Gametângios conjuntos, sejuntos, na base das (1 $\left.1^{\mathrm{a}}-\right) 2^{\mathrm{a}}-3^{\mathrm{a}}$-furcação. Núculas 1-(2-3) por nó, 226-607 $\mu \mathrm{m}$ compr. $\times 170-405 \mu \mathrm{m}$ diâm., convoluções 6-7(-8), corônula 37-48,6 $\mu \mathrm{m}$ compr. $\times$ 59,5-83 $\mu \mathrm{m}$ diâm. Oósporos $238 \mu \mathrm{m}$ compr. $\times 246 \mu \mathrm{m}$ diâm., estrias 5, fossa $50 \mu \mathrm{m}$ larg. Glóbulos (121,5-)218-284(-358) ㅆm diâm., escudos 8 , triangulares.
Primeiro registro da espécie para o Estado do Mato Grosso, tendo sido citada para Mato Grosso do Sul por Bueno \& Bicudo (1997), como Nitella furcata (Roxburgh ex Bruzelius) C. Agardh emend R.D. Wood subsp. Alagellifera (J. Groves \& G.O. Allen) R.D. Wood.

Tanto o limite métrico superior do comprimento $(607 \mu \mathrm{m})$ quanto o inferior $(170 \mu \mathrm{m})$ do diâmetro da núcula foram atualmente ampliados quando comparados aos valores em Picelli-Vicentim et al. (2004). Os espécimes da unidade amostral SP104176 ampliaram o limite métrico do diâmetro do glóbulo (121,5-358 $\mu \mathrm{m})$, quando comparado com Picelli-Vicentim et al. (2004).

Distribuição no Brasil: [= N. furcata (Roxburgh ex Bruzelius) C. Agardh emend. R.D. Wood subsp. flagellifera (J. Groves \& G.O. Allen) R.D. Wood]. Mato Grosso do Sul (Bueno \& Bicudo 1997), São Paulo (Picelli-Vicentim 1990, Vieira Junior et al. 2002, Picelli-Vicentim et al. 2004), Rio Grande do Sul (Prado 2003).

Material examinado: BRASIL. Mato Grosso: Barra do Garças, 30-V-1968, D.M. Vital s.n. (SP114482); Xavantina, 30-V-1968, D.M. Vital s.n. (SP104176). Mato Grosso do Sul: Corumbá, 21-VIII-1991, N.C. Bueno 245 (CPAP8311); 21-VIII-1991, N.C. Bueno 248 (CPAP8314); Miranda, 6-VI-1973, D.M. Vital 2322 (SP116381).

Nitella gollmeriana A. Braun, Monat. der Deutschen Akad. der Wiss. zu Berlin: 355. 1858.

Figuras $2 \mathrm{~J}-\mathrm{R}$

Plantas monóicas, 14 cm alt., incrustação calcárea ausente. Caulóide 652,5-1305 um diâm. Entrenós mais longos que os râmulos, $0,8-2,3 \mathrm{~cm}$ compr. Râmulos estéreis bem desenvolvidos, 7-8, 1-furcados, 1,3-1,8 cm compr. $\times 526-736 \mu \mathrm{m}$ diâm., 1-furcados; raios primários 5-8, 1-1,2 cm compr., constituindo a maior parte do râmulo; dáctilos 2-4, 1-celulados, ápices acuminados, 1263-3010(-5660) $\mu \mathrm{m}$ compr. $\times 147-315 \mu \mathrm{m}$ diâm. Râmulos férteis reduzidos em capítulos, 5-7, 1-6(-7) mm compr. $\times 136,5-293$ m diâm., 1-furcados; dáctilos 2-3, 1-celulados, acuminados, geralmente abreviados, 568-990 $\mu \mathrm{m}$ compr. $\times 105-252 \mu \mathrm{m}$ diâm. Capítulos numerosos, 1-3 por verticilo, axilares, estipitados, semi-esféricos a esféricos, condensados, sem muco, 1-4 mm diâm. Gametângios conjuntos. Núculas sésseis, 333-466,5 $\mu \mathrm{m}$ compr. $\times 266,6-$ 358,2 $\mu \mathrm{m}$ diâm., convoluções 8, corônula decídua, 


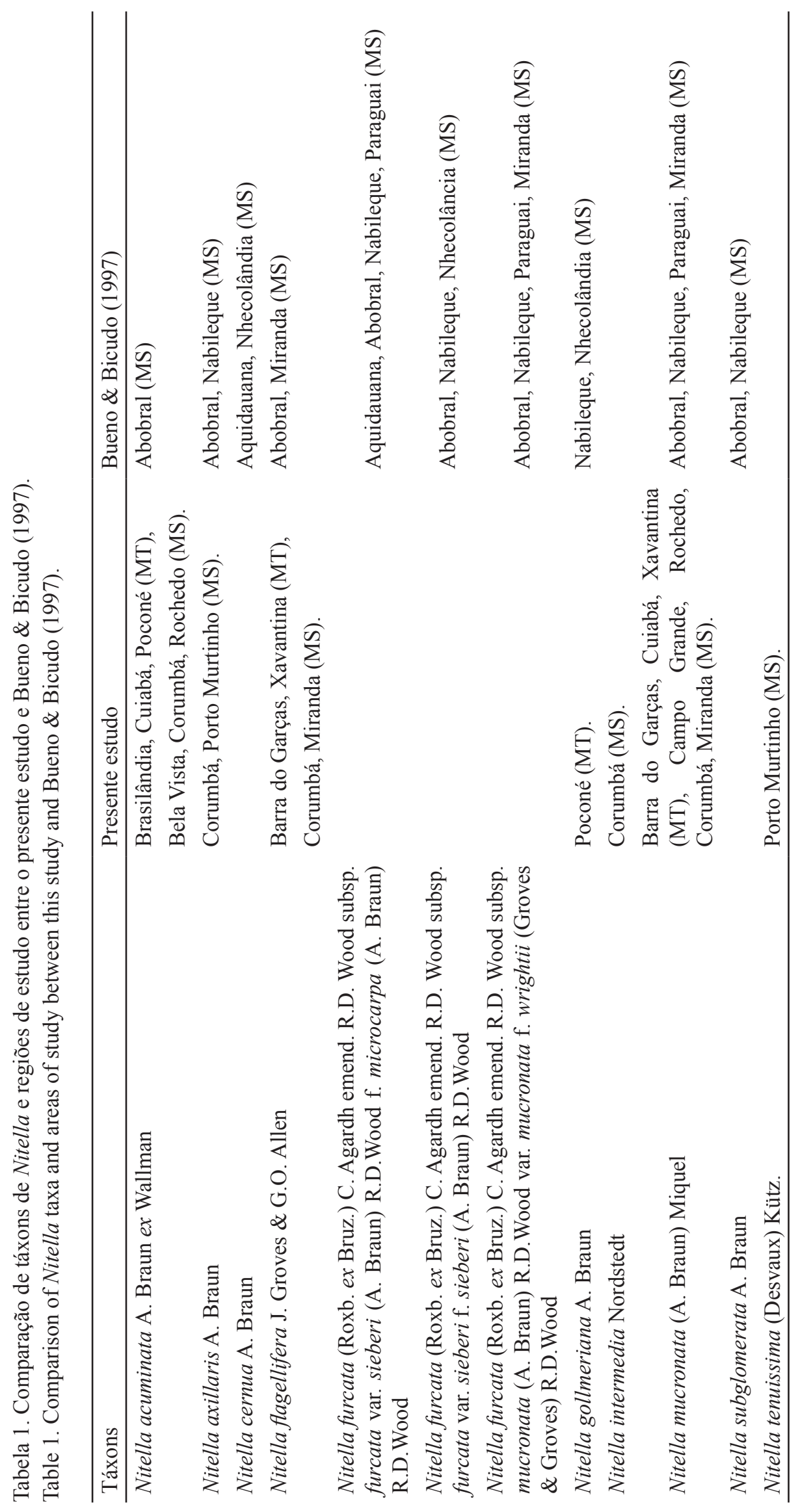



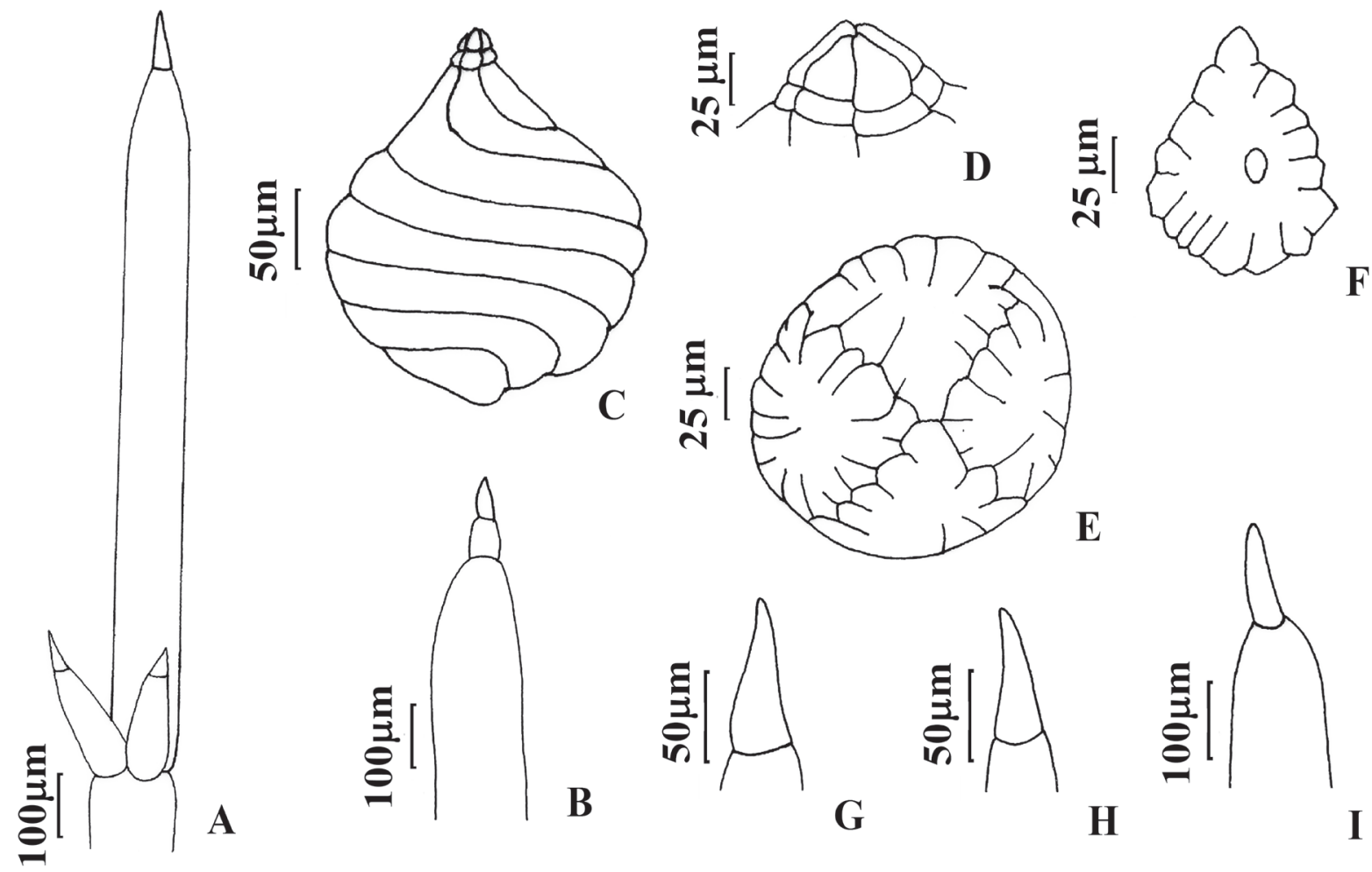

\section{F}

A

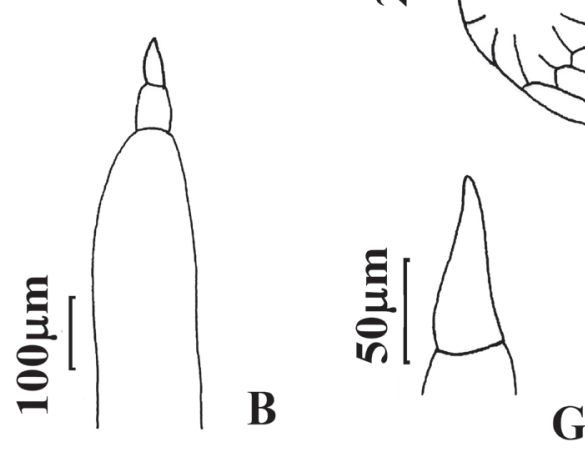

G $\quad$ H
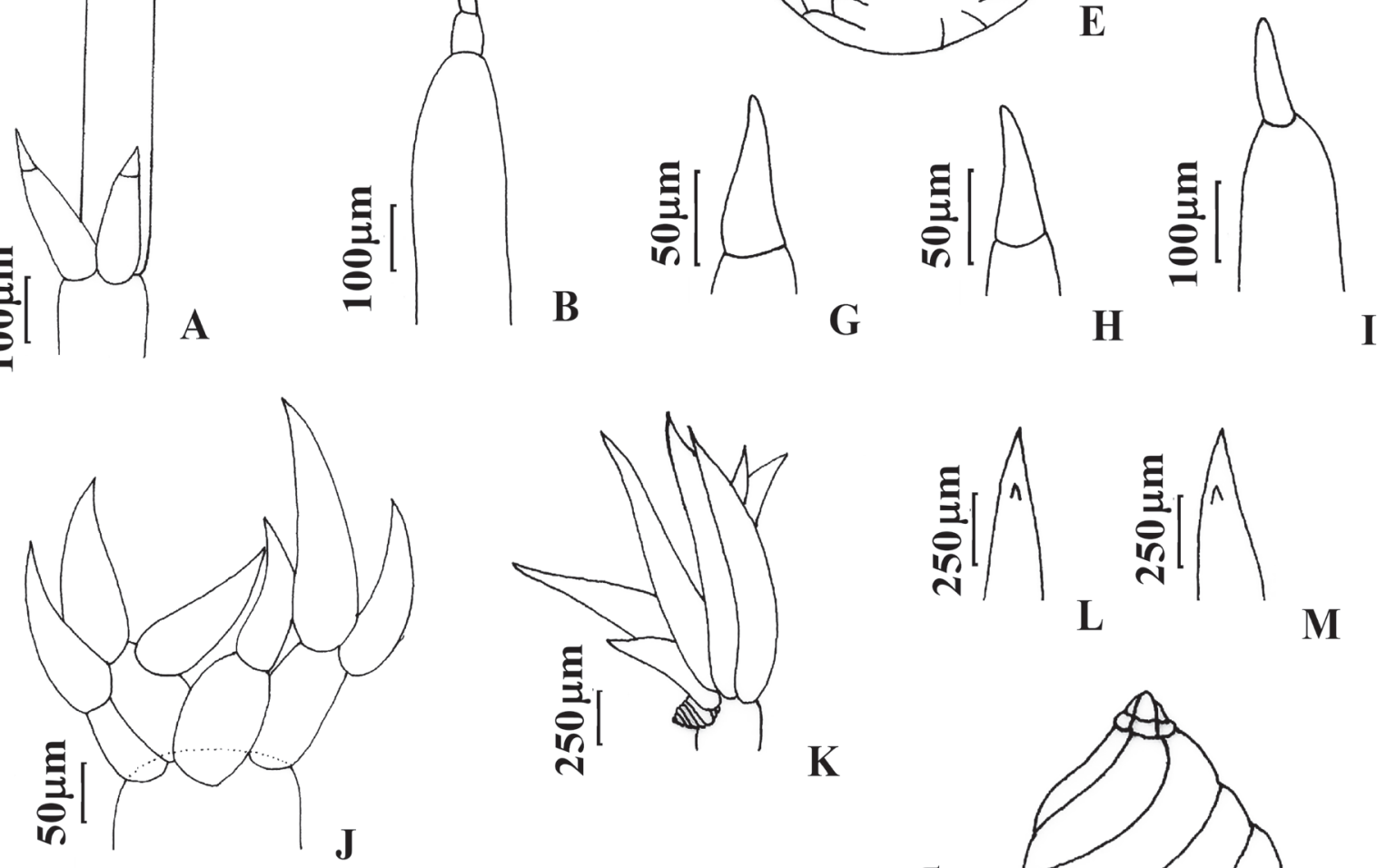

$\mathbf{E}$
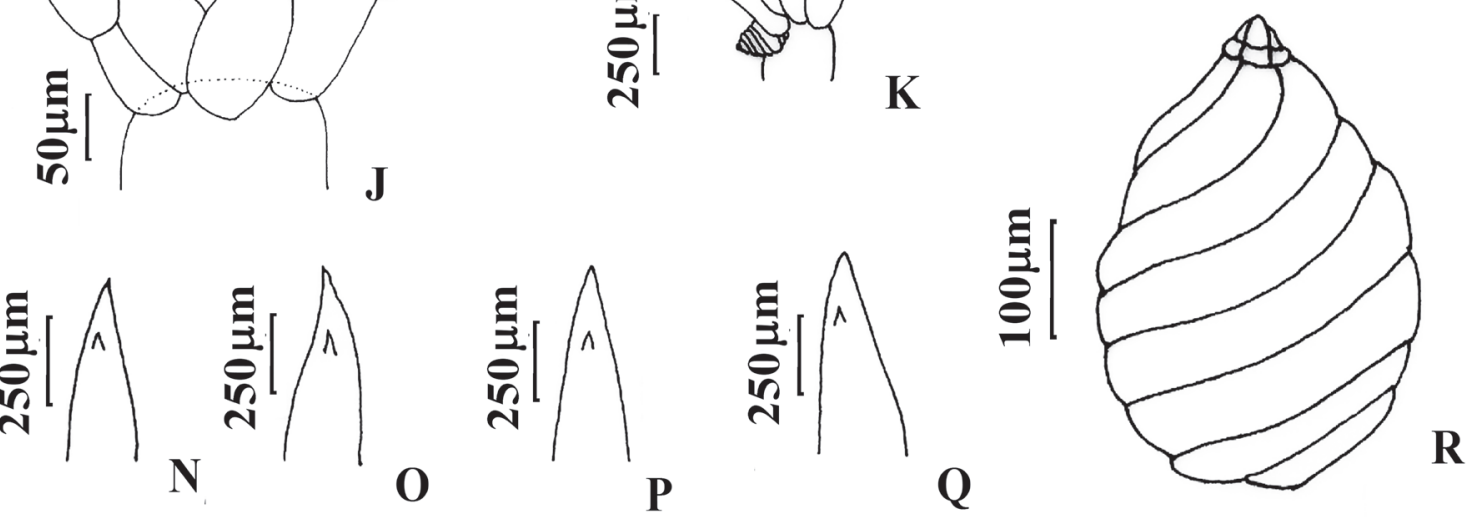

Figura 2. Espécies de Nitella do Mato Grosso e Mato Grosso do Sul, Brasil. A-I. Nitella flagellifera. A-B. Dáctilos 2-celulados. C. Núcula. D. Corônula. E. Glóbulo. F. Escudo triangular. G-I. Ápice dos dáctilos. J-R. Nitella gollmeriana. J. Râmulo verticilado estéril. K. Ápice de râmulo fértil. L-Q. Dáctilos 1-celulados. R. Núcula.

Figure 2. Nitella species of Mato Grosso and Mato Grosso do Sul, Brazil. A-I. Nitella flagellifera. A-B. 2-celled dactyls. C. Oogonium. D. Coronula. E. Antheridium. F. Triangular scute. G-I. Dactyl apices. J-R. Nitella gollmeriana. J. Sterile whorl. K. Fertile branchlets apices. L-Q. 1-celled dactyls. R. Oogonium. 
47-51 $\mu \mathrm{m}$ compr. $\times 53-9,5 \mu \mathrm{m}$ diâm. Oósporos 291,5-308,2 $\mu \mathrm{m}$ compr. $\times$ 254-291,5 $\mu \mathrm{m}$ diâm., estrias 5-7, fossa 44-58 $\mu \mathrm{m}$ larg., parede do oósporo finamente granulada. Glóbulos 191,5-252,6 بm diâm., escudos 8 , triangulares.

Primeiro registro da espécie para o Estado de Mato Grosso. Em relação aos espécimes examinados no presente trabalho, alguns gametângios femininos apresentaram corônula decídua (CPAP18677), concordando com uma das características diagnósticas da espécie segundo Wood \& Imahori (1965). No presente trabalho, as variações métricas ampliaram os limites do comprimento do râmulo verticilado fértil de 1-2 mm para 1-6(-7) $\mathrm{mm}$. A unidade amostral CPAP10411 ampliou os limites inferiores do comprimento $(333 \mu \mathrm{m})$ e do diâmetro $(266,6 \mu \mathrm{m})$ da núcula e os maiores valores do comprimento dos dáctilos $(5660 \mu \mathrm{m})$, quando comparados com Picelli-Vicentim et al. (2004).

Distribuição no Brasil: Mato Grosso do Sul (Bueno \& Bicudo 1997), São Paulo (Bicudo 1969, Bicudo \& Yamaoka 1978, Picelli-Vicentim 1990, Branco \& Necchi Junior 1996, Necchi Junior et al. 2000, Vieira Junior et al. 2002, Picelli-Vicentim et al. 2004), Rio Grande do Sul (Prado 2003).

Material examinado: BRASIL. Mato Grosso: Poconé, 14-IV-1993, V.J. Pott 1882 (CPAP10411); 30-X-1999, V.J. Pott 4083 (CPAP18677).

Nitella intermedia Nordstedt in T.F. Allen, Char. Amer. Exs. no. 2. 1880.

Figuras $3 \mathrm{~A}-\mathrm{F}$

Plantas monóicas, $8 \mathrm{~cm}$ alt., incrustação calcárea ausente. Caulóide 568-799 $\mu \mathrm{m}$ diâm. Entrenós menores que o comprimento dos râmulos, $0,1-0,3 \mathrm{~cm}$ compr. Râmulos estéreis 5-7, até $2 \mathrm{~cm}$ compr., 2-3 furcados, raios primários 5-6, 0,3-1 cm compr. $\times 157-210,5 \mu$ m diâm., 0,5 vezes o compr. dos râmulos, secundários 6-7, sendo 1 geralmente central, terciários 3-4, quaternários 2-3 (quando presentes), dáctilos 3-5, 2-3-celulados, 2610-4041(-5473) $\mu \mathrm{m}$ compr. $\times$ 252-399 $\mu \mathrm{m}$ diâm. Râmulos férteis: 6-7, 0,4-1,6 cm compr., 2-3-furcados, às vezes compactados em capítulos; dáctilos 3-5, 2-3celulados, geralmente alongados, ocasionalmente abreviados, 399-616 $\mu \mathrm{m}$ compr. $\times 108-174 \mu \mathrm{m}$ diâm. Capítulos obscuros, verticilos superiores férteis e levemente reduzidos, 27-33 mm diâm., com muco. Gametângios conjuntos, freqüentemente no segundo nó, pedunculados. Pedúnculos 26,5-58(-110,5) $\mu \mathrm{m}$ compr. $\times$ 39-100 $\mu \mathrm{m}$ diâm. Núculas 308-500 $\mu \mathrm{m}$ compr. $\times$ 258-382,5 $\mu \mathrm{m}$ diâm., corônula $39 \mu \mathrm{m}$ compr. × 39-53 $\mu \mathrm{m}$ diâm., convoluções 7-9. Oósporos 291,5-500 $\mu \mathrm{m}$ compr. $\times$ 325-375 $\mu \mathrm{m}$ diâm., estrias 5-8, fossa $50 \mu \mathrm{m}$ compr., parede do oósporo granulada a papilada. Glóbulos 175-283 $\mu \mathrm{m}$ diâm., escudos 8, triangulares.

Primeiro registro da espécie para o Estado de Mato Grosso do Sul e Brasil [= Nitella gracilis (Smith) C. Agardh emend. R.D. Wood subsp. gracilis var. gracilis f. intermedia (Nordstedt) R.D. Wood].

Nitella gracilis (Smith) C. Agardh emend. R.D. Wood subsp. gracilis var. gracilis $\mathrm{f}$. gracilis (tipo) e Nitella gracilis (Smith) C. Agardh emend. R.D. Wood subsp. gracilis var. havaiensis (Nordstedt) R.D. Wood foram citadas pela primeira vez para o Estado do Rio Grande do Sul por Astorino (1983); N. gracilis (Smith) C. Agardh emend. R.D. Wood foi citada para o mesmo Estado por Prado (2003).

De acordo com Wood \& Imahori (1965), $N$. intermedia é uma forma taxonômica de $N$. gracilis (Smith) C. Agardh. A identificação da espécie como $N$. intermedia Nordstedt deu-se em função da presença dos dáctilos 2-3-celulados, alongados, célula terminal não mucronada, confluente com a penúltima célula dos dáctilos.

$\mathrm{O}$ material atualmente analisado registrou a presença de um pequeno pedúnculo na base das núculas $[26,5-58(-110,5) \times 39-100 \mu \mathrm{m}]$. A condição dos glóbulos da espécie semelhante $N$. gracilis (Smith) C. Agardh emend. R.D. Wood é tetra-escudada, porém, em $N$. intermedia Nordstedt os glóbulos são octa-escudados como registrado no presente material. Distribuição no Brasil: nada consta.

Material examinado: BRASIL. Mato Grosso do Sul: Corumbá, 30-VI-1992, N.C. Bueno 446 (CPAP10210).

Nitella mucronata (A. Braun) Miquel in H.C. van Hall, Flora Belgii Septentrionalis 2: 428. 1840. Figuras 3 G-J

Plantas monóicas, $10-15 \mathrm{~cm}$ alt., incrustação calcárea ausente. Entrenós 1-3 cm compr., 1-2 vezes o comprimento do râmulo verticilado. Caulóide 399-758 $\mu \mathrm{m}$ diâm. Râmulos 5-7, 0,5-1,6 cm compr. $\times 370 \mu \mathrm{m}$ diâm., 2-3-furcados. Raios primários 5-7, 4-5 mm compr. $\times$ 230-672,4 $\mu$ m diâm., raios secundários 4-7, raios terciários 2-4, raios quaternários 2-4. Dáctilos 3, 2(-3)-celulados, 133-1863(-2625) $\mu \mathrm{m}$ 
compr. $\times$ 58-155 $\mu \mathrm{m}$ diâm., alongados, ápice agudo. Râmulos férteis podem ser reduzidos, formando ocasionalmente 3-5 capítulos por verticilo, ca. 1,5 mm diâm. Gametângios conjuntos ou sejuntos, na base de todas as furcações. Núculas 1-(2) por nó, geralmente ausente no primeiro nó, 204-662 $\mu \mathrm{m}$ compr. $\times$ 132,60-469 $\mu \mathrm{m}$ diâm., convoluções 8-10, corônula 41-55 $\mu$ mcompr. $\times 52-75,48 \mu$ m diâm. Oósporos 157-366 $\mu \mathrm{m}$ compr. $\times 149-301 \mu \mathrm{m}$ diâm., parede do oósporo reticulada. Glóbulos (128-)141-469,5 $\mu \mathrm{m}$ diâm., escudos 8, triangulares.

Primeira citação da ocorrência de Nitella mucronata (A. Braun) Miquel [= N. furcata (Roxburgh ex Bruzelius) C. Agardh emend. R.D. Wood subsp. mucronata (A. Braun) R.D. Wood var. mucronata f. mucronata] para o Estado de Mato Grosso. Os espécimes de N. mucronata (A. Braun) Miquel apresentam râmulos férteis divididos 2 ou 3 vezes, formam ocasionalmente densos capítulos e apresentam dáctilos predominantemente alongados, variando de 2 a 3-celulados, com células mucronadas e agudas (Urbaniak 2009) e os oósporos possuem ornamentação reticulada (Caisová et al. 2008). Os espécimes ora analisados concordaram com a descrição acima, apoiando-se também nos trabalhos de Corillion (1975), Bueno \& Bicudo (1997), Picelli-Vicentim \& Bicudo (1993) e Picelli-Vicentim et al. (2004).

Migula (1897) e Allen (1928) ressaltaram a condição polimórfica da espécie $N$. mucronata, tratadas por Wood \& Imahori (1965) como formas taxonômicas. Estudos recentes (Casanova 2005, 2009, Sakayama 2008) reconhecem que a controversa classificação de Wood \& Imahori $(1964,1965)$ baseia-se em conceito errôneo de espécie, desta forma, o tratamento adotado neste trabalho apóia-se em uma classificação natural dos grupos.

Distribuição no Brasil: [= Nitella furcata (Roxburgh ex Bruzelius) C. Agardh emend. R.D. Wood subsp. mucronata (A. Braun) R.D. Wood var. mucronata f. mucronata]. Ceará (Wood \& Imahori 1965), Mato Grosso do Sul (Bueno \& Bicudo 1997), São Paulo (Picelli-Vicentim 1990, Necchi Junior et al. 2000, Vieira Junior et al. 2002, Picelli-Vicentim et al. 2004, Branco \& Necchi Junior 1998).

Material examinado: BRASIL. Mato Grosso: Barra do Garças, 30-V-1968, D.M. Vital s.n. (SP104176); Cuiabá, 17-VI-1969 D.M. Vital s.n. (SP104151); Xavantina, 12-VI-1969, D.M. Vital S.n. (SP104153). Mato Grosso Do Sul: Campo Grande, 30-I-1979, L.N.C. Rodrigues s.n. (SP152602); Campo Grande,
20-I-1979, R.M.T. Bicudo \& A.G. Pedrini s.n. (SP152604); Rochedo, 25-I-1979, M.R.A. Braga \& V.L.M. Hussar s.n. (SP152603), Corumbá, 6-VII-1894, G.A.N. Malme s.n. (SP114569); Miranda, 9-VI-1973, D.M. Vital 2347 (SP116384).

Nitella tenuissima (Desvaux) Kütz., Phyc. Generalis: 319. 1843.

Figuras $3 \mathrm{~K}-\mathrm{Q}$

Plantas monóicas, pequenas e delicadas, $10 \mathrm{~cm}$ alt. Caulóide 216,5-358 $\mu \mathrm{m}$ diâm. Entrenós 0,8-2 vezes o tamanho dos râmulos, 0,9-2 cm compr. Râmulos monomórficos, furcados, podendo o fértil ser maior que $1 \mathrm{~cm}$; 5-8, 2-3-furcados; raios primários $5,0.3$ vezes o comprimento dos râmulos, $80-110 \mu \mathrm{m}$ diâm., raios secundários 5-7, dos quais 1 é central, terciários 3-7, quaternários 4, dáctilos 3-5, 2-celulados, células terminais longas e estreitas, 291,5-1199 $\mu \mathrm{m}$ compr. $\times 33-84 \mu$ m diâm. Capítulos ausentes, verticilos superiores ocasionalmente compactos. Gametângios conjuntos, sejuntos, ausentes no primeiro nó. Núculas 308-366,5 $\mu \mathrm{m}$ compr. $\times 216,5-241,5 \mu \mathrm{m}$ diâm., corônula 33-50 $\mu \mathrm{m}$ compr. $\times 50-59 \mu \mathrm{m}$ diâm., convoluções (7-)9-10. Oósporos 200-216,58 $\mu \mathrm{m}$ compr. $\times$ 175-191,6 diâm., estrias 7-8, fossa 30-44 $\mu \mathrm{m}$ larg., parede do oósporo reticulada. Glóbulos 150-166,6 $\mu \mathrm{m}$ diâm., curto estipitados. Pedúnculo 16-45 $\mu \mathrm{m}$ compr.

Primeiro registro da ocorrência de $N$. tenuissima (Desvaux) Kützing para o Estado de Mato Grosso do Sul. A identificação da espécie baseou-se na análise de apenas uma amostra. Nittela tenuissima (Desvaux) Kützing caracteriza-se por ser monóica, ter râmulos verticilados com 2-4 furcações, dáctilos 2 (-3)-celulados com célula terminal cônica, ausência de capítulos, presença de verticilos compactos, parede do oósporo reticulada e gametângios ausentes no primeiro nó dos râmulos verticilados.

Nitella tenuissima (Desvaux) Kützing é muito semelhante a $N$. gracilis (Smith) C. Agardh. Porém, N. gracilis raramente possui râmulo secundário central e a parede do oósporo pode ser granulada, papilada ou porosa, mas nunca reticulada. Optou-se pela identificação do material HMS10937 como $N$. tenuissima (Desvaux) Kützing em função da ausência dos gametângios no nó basal dos râmulos e da presença de raio secundário central e de dáctilos 2 -celulados. Foram registrados ainda dáctilos 3-celulados, característica rara desta espécie. A unidade 

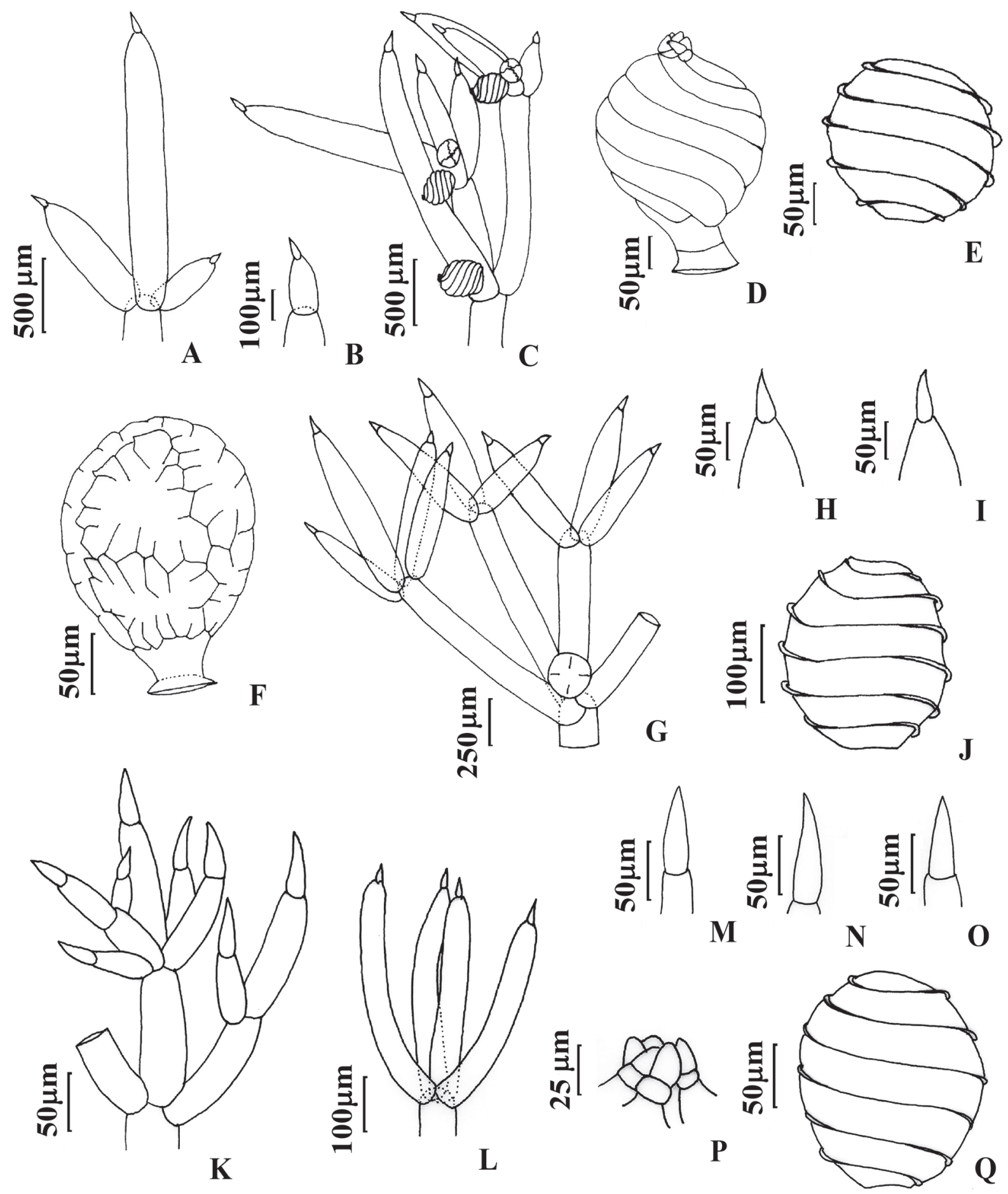

Figura 3. Espécies de Nitella do Mato Grosso e Mato Grosso do Sul, Brasil. A-F. Nitella intermedia. A-B. Dáctilos 2-3-celulados. C. Râmulo verticilado fértil. D. Núcula pedunculada. E. Oósporo. F. Glóbulo pedunculado. G-J. Nitella mucronata. G. Râmulo verticilado estéril. H-I. Ápice dos dáctilos. J. Oósporo. K-Q. Nitella tenuissima. K-L. Râmulo verticilado estéril. M-O. Dáctilos 2-celulados. P. Núcula. Q. Oósporo.

Figure 3. Nitella species of Mato Grosso and Mato Grosso do Sul, Brazil. A-F. Nitella intermedia. A-B. 2-3-celled dactyls . C. Fertile whorl. D. Stipitate oogonium. E. Oospore. F. Stipitate antheridium. G-J. Nitella mucronata. G. Sterile whorl. H-I. Dactyls apices. J. Oospore. K-Q. Nitella tenuissima. K-L. Sterile whorl. M-O. 2-celled dactyls. P. Oogonium. Q. Oospore. 
amostral HMS10937 inclui glóbulos curto-estipitados, com pedúnculo de 16-45 $\mu \mathrm{m}$ comprimento.

Distribuição no Brasil: Rio Grande do Sul (Astorino 1983).

Material examinado: BRASIL. Mato Grosso do Sul: Porto Murtinho, 7-IX-2005, V.J. Pott 8145 (HMS10937).

\section{Discussão}

O presente trabalho apresenta sete novas citações do gênero Nitella para os Estados do Mato Grosso e Mato Grosso do Sul. As espécies N. acuminata, $N$. flagellifera, N. gollmeriana e N. mucronata são novas citações para o Estado do Mato Grosso, enquanto $N$. intermedia e $N$. tenuissima são pioneiramente registradas para o Estado do Mato Grosso do Sul (tabela 1).

A espécie que apresentou maior distribuição geográfica foi $N$. mucronata, ocorrendo nos municípios de Barra do Garças, Cuiabá e Xavantina (Estado do Mato Grosso), e nos municípios de Campo Grande, Corumbá, Miranda e Rochedo (Estado do Mato Grosso do Sul). Nitella acuminata foi registrada para os municípios Brasilândia, Cuiabá e Poconé (Estado do Mato Grosso) e Bela Vista, Corumbá e Rochedo (Estado do Mato Grosso do Sul).

Em relação ao material analisado, $N$. acuminata é a espécie mais amplamente distribuída em território nacional. Ocorre em cinco estados brasileiros, são eles: Mato Grosso do Sul (Bueno \& Bicudo 1997), Paraná (Thomaz et al. 2003), Rio de Janeiro (Bicudo 1969, Bicudo \& Yamaoka 1978), São Paulo (Picelli-Vicentim 1990, Bicudo 1969, Bicudo \& Yamaoka 1978, PicelliVicentim \& Bicudo 1993, Picelli-Vicentim et al. 2004) e Rio Grande do Sul (Prado 2003). A espécie $N$. tenuissima apresentou distribuição geográfica restrita, até o momento no Estado do Rio Grande do Sul (Astorino 1983), enquanto $N$. intermedia consta no presente trabalho seu primeiro registro no país.

As variações morfométricas das espécies podem representar variações entre as populações analisadas ou demonstrar plasticidade fenotípica dos espécimes estudados. Tais variações podem estar relacionadas às diferentes características ambientais, tais como temperatura, variação do nível da água, correnteza e profundidade, sob as quais estes espécimes se desenvolveram (Casanova 1994, Vieira Junior \& Necchi Junior 2002, Asaeda et al. 2007,
Blindow \& Schütte 2007). Estudos filogenéticos concluídos recentemente são contribuições efetivas ao entendimento da diversidade de Nitella (Karol et al. 2001, Sakayama et al. 2002, 2004, Sanders et al. 2003, Sakayama 2008).

O presente estudo carece de informações a respeito de fatores ambientais devido à ausência de informações nas fichas dos materiais provenientes de empréstimos de herbários do país. Enfatizamos que o registro de informações físicas e químicas dos locais de amostragem podem ser úteis na busca por possíveis correlações que expliquem as variações métricas observadas nos materiais estudados e sugerimos o registro de tais fatores em amostragens futuras.

De acordo com Urbaniak (2009), o estudo das características interespecíficas da ornamentação da parede do oósporo de Characeae pode ser um importante caráter taxonômico, em especial para o gênero Nitella. No presente trabalho, N. acuminata e $N$. gollmeriana apresentaram a ornamentação da parede celular do oósporo finamente granulada; $N$. intermedia apresentou parede variando de granulada a papilada e $N$. mucronata e $N$. tenuissima apresentaram parede reticulada.

\section{Agradecimentos}

CEMB agradece ao CNPq, Conselho Nacional de Desenvolvimento Científico e Tecnológico, por Bolsa de Produtividade CNPq - Nível 1A. TM agradece a Fundação Parque Tecnológico Itaipu, por bolsa nível Mestrado.

\section{Literatura citada}

Allen, G.O. 1928. Charophyte notes from Sahanpur, U. P. - Journal of Indian Botanical Society 7: 46-69.

Asaeda, T., Rajapakse, L. \& Sanderson, B. 2007. Morphological and reproductive acclimations to growth of two charophyte species in shallow and deep water. Aquatic Botany 86: 393-401.

Astorino, H.A.B. 1983. Charophyceae do Estado do Rio Grande do Sul: uma contribuição ao seu inventário. Dissertação de Mestrado, Universidade Estadual Paulista "Júlio de Mesquita Filho", Rio Claro.

Bicudo, C.E.M. \& Menezes, M. 2006. Gêneros de algas de águas continentais do Brasil. Editora RiMa, São Paulo.

Bicudo, R.M.T. 1969. Brazilian Characeae of the herbarium of the Instituto de Botânica, São Paulo. Nova Hedwigia 17: 1-17.

Bicudo, R.M.T. 1974. O gênero Chara (Charophyceae) no Brasil, 1: Subseção Willdenowia R.D. Wood. Rickia 6: 127-189. 
Bicudo, R.M.T. 1977. O gênero Chara (Charophyceae) no Brasil, 2: Seção Chara. In: Anais do XXVI Congresso Nacional de Botânica. Sociedade Botânica do Brasil, Rio de Janeiro.

Bicudo, R.M.T. 1979. O gênero Chara (Charophyceae) no Brasil, 3: Seção Charopsis (Kützing emend. Rupr., Leonh.) R.D. Wood. Rickia 8: 17-26.

Bicudo, R.M.T. \& Yamaoka, D.M. 1978. O gênero Nitella (Charophyceae) no Brasil, 1: subgênero Nitella. Acta Biologica Paranaense 7: 77-98.

Blindow, I. \& Schütte, M. 2007. Elongation and mat formation of Chara aspera under different light and salinity conditions. Hydrobiologia 584: 69-76.

Braun, A. \& Nordstedt, C.F.O. 1882. Fragmente einer Monographie der Characeen: nach den hinterlassenen Manuscripten. A Braun's herausgegeben von Dr. Otto Nordstedt. Abhandlungen der Königinen Akademie der Wissenschaftlichen Berlim 1882: 1-211.

Bueno, N.C. \& Bicudo, C.E.M. 1997. Characeae (Charophyceae) do Pantanal de Mato Grosso do Sul, Brasil: Nitella. Hoehnea 24: 29-55.

Bueno, N.C. \& M. Bicudo, C.E.M. 2006. Temporal variation of Nitella furcata subsp. mucronata var. mucronata f. oligospira (Charophyceae) in the Ninféias pond, São Paulo State, southeast Brazil. Acta Botanica Brasilica 20: 1-11.

Bueno, N.C., Bicudo, C.E.M., Biolo, S. \& Meurer, T. 2009. Levantamento taxonômico das Characeae (Chlorophyta) de Mato Grosso e Mato Grosso do Sul, Brasil: Chara. Revista Brasileira de Botânica 32: 735-750.

Branco, C.C.Z. \& Necchi Junior, O. 1996. Survey of stream macroalgae of eastern Atlantic Rainforest of São Paulo State, southeastern Brazil. Algological Studies 80: 35-57.

Branco, L.H.Z \& Necchi Junior, O. 1998. Distribution of macroalgae in three tropical drainage basins of Southeastern Brazil. Archiv fur Hydrobiologie 142: 241-256.

Caisová, L., Husak, S. \& Komárek, J. 2008. Nitella mucronata (Br.) Miquel (Charophyta) in the Czech Republic. Fottea 8: 105-107.

Casanova, M.T. 1994. Vegetative and reproductive responses of charophytes to water-level fluctuations in permanent and temporary wetlands in Australia. Australian Journal of Marine and Freshwater Research 45: 1409-1419.

Casanova, M.T. 2005. An overview of Chara L. in Australia (Characeae, Chlorophyta). Australian Systematic Botany 18: 25-39.

Casanova, M.T. 2009. An overview of Nitella (Characeae, Charophyceae) in Australia. Australian Systematic Botany 22: 193-218.
Corillion, R. 1975. Flore des Charophytes (Characées) du Massif Armoricain et des contrées voisines d'Europe occidentale. In: I.V.Tome (ed.). Flore et vegetation du Massif Armoricain Jouve Éditeurs, Paris, pp. 214.

Groves, H. \& Groves, J. 1911. Characeae. In: I. Urban (ed.). Symbolae Antillanae, seu Fundamenta Flora Indiae. Occidentalis 7: 30-44.

John, D.M. \& Moore, J.A. 1987. A SEM study of the oospore of some Nitella species (Chlorophyta, Charales) with descriptions of wall ornamentation and an assessement of its taxonomic importance. Phycologia 26: 334-355.

Karol, K.G., McCourt, R.M., Cimino, M.T., \& Delwiche, C.F. 2001. The closest living relatives of land plants. Science 294: 2351.

Krause, W. 1997. Charales (Charophyceae). In: H. Ettl, G. Gärtner, H. Heyning and D. Molenhauser (eds.). Sußwasserflora von Mitteleuropa. vol. 18. Gustav Fischer, Sttutgart.

Migula, W. 1897. Die Characeen. In: L. Rabenhorst (ed.). Kryptogamenflora von Deutschland, Österreich und der Schweiz. Kummer, Leipzig, pp. 1-765.

Moore, J.A. 1986. Charophytes of Great Britain and Ireland. Botanical Society of the British Isles, London.

Necchi Junior, O., Branco, C.C.Z. \& Branco, L.H.Z. 2000. Distribution of stream macroalgae in São Paulo State, southeastern Brazil. Algological Studies 97: 43-57.

Picelli-Vicentim, M.M. 1990. Characeae do Estado de São Paulo: inventário sistemático. Tese de Doutorado, Universidade Estadual Paulista "Júlio de Mesquita Filho", Rio Claro.

Picelli-Vicentim, M.M. \& Bicudo, C.E.M. 1993. Criptógamos do Parque estadual das Fontes do Ipiranga, São Paulo, SP. Algas, 4: Charophyceae. Hoehnea 20: 9-22.

Picelli-Vicentim, M.M., Bicudo, C.E.M. \& Bueno, N.C. 2004. Flora ficológica do Estado de São Paulo, 5: Charophyceae. RiMa Editora, São Carlos.

Pott, V.J., Bueno, N.C. \& Silva, M.P. 1992. Levantamento florístico e fitossociológico de macrófitas aquáticas em lagoas da Fazenda Leque, Pantanal, MS. In: Anais do VIII Congresso da Sociedade Botânica de São Paulo. Sociedade Botânica de São Paulo, Campinas, pp. 91-99.

Prado, J.F. 2003. Characeae do Rio Grande do Sul, Brasil. Tese de Doutorado,Universidade Federal do Rio Grande do Sul, Porto Alegre.

Proctor, V.W., Griffin, D.G. \& Hotchkiss, A.T. 1971. A sinopsis of the genus Chara, series Gymnobasalia (subsection Willdenowia R.D. Wood). American Journal of Botany 58: 894-901.

Sakayama, H. 2008. Taxonomy of Nitella (Charales, Charophyceae) based on comparative morphology of oospore and multiple DNA marker phylogeny using cultured material. Phycological Research 56: 202-215. 
Sakayama, H., Hara, Y. \& Nozaki, H. 2004. Taxonomic re-examination of Nitella (Charales, Charophyceae) from Asia, and phylogenetic relationship within the genus based on rbcL and atpB gene sequences. Phycologia 43: 91-104.

Sakayama, H., Nozaki, H., Kasaki, H. \& Hara, Y. 2002. Taxonomic re-examination of Nitella (Charales, Charophyceae) from Japan, based on microscopical studies of oospores wall ornamentation and $\mathrm{rbcL}$ gene sequences. Phycologia 41: 397-408.

Sanders, E.R., Karol, K.G. \& McCourt. R.M. 2003. Ocurrence of $m a t K$ in a $\operatorname{trn} K$ group II intron in charophyte green algae and phylogeny of the Characeae. American Journal of Botany 90: 628-633.

Thomaz, S.M., Souza, D.C. \& Bini, L.M. 2003. Species richness and beta diversity of aquatic macrophytes in a large subtropical reservoir (Itaipu Reservoir, Brazil): the influence of limnology and morphometry. Hydrobiologia 505: 119-128.

Urbaniak, J. 2009. Oospore variation in Nitella gracilis and Nitella mucronata (Charales, Charophyceae) from Poland. Biologia 64: 252-260.
Vieira Junior, J., Necchi Junior, O., Branco, C.C.Z. \& Branco, L.H.Z. 2002. Characeae (Chlorophyta) em ecossistemas lóticos do Estado de São Paulo, Brasil: Gênero Nitella. Hoehnea 29: 249-266.

Vieira Junior, J., Necchi Junior, O., Branco, C.C.Z. \& Branco, L.H.Z. 2003. Characeae (Chlorophyta) em ecossistemas lóticos do Estado de São Paulo, Brasil: gênero Chara e distribuição ecológica. Hoehnea 30: 53-70.

Vieira Junior, J. \& Necchi Junior, O. 2002. Microhabitat and plant structure of Characeae (Chlorophyta) populations in streams from São Paulo State, southeastern Brazil. Cryptogamie Algologie 23: 51-63.

Wood, R.D. \& Imahori, K. 1964. A revision of the Characeae, 2: iconograph of the Characeae. J. Cramer, Weinhen.

Wood, R.D. \& Imahori, K. 1965. A revision of the Characeae, 1: monograph of the Characeae. J. Cramer, Weinhen. 